\title{
The influence of orthopedic corsets on the incidence of pathological fractures in patients with spinal bone metastases after radiotherapy
}

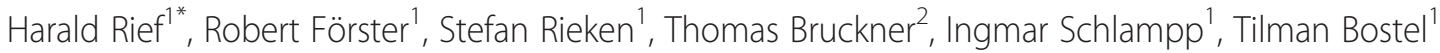 \\ and Jürgen Debus ${ }^{1}$
}

\begin{abstract}
Background: Clinical care of unstable spinal bone metastases in many centers often includes patient immobilization by means of an orthopedic corset in order to prevent pathological fractures. The aim of this retrospective analysis was to evaluate the incidence of pathological fractures after radiotherapy (RT) in patients with and without orthopedic corsets and to assess prognostic factors for pathological fractures in patients with spinal bone metastases.

Methods: The incidence of pathological fractures in 915 patients with 2.195 osteolytic metastases in the thoracic and lumbar spine was evaluated retrospectively on the basis of computed tomography (CT) scans between January 2000 and January 2012 depending on prescription and wearing of patient-customized orthopedic corsets.

Results: In the corset group, 6.8 and $8.0 \%$ in no-corset group showed pathological fractures prior to RT, no significant difference between groups was detected ( $p=0.473$ ). After 6 months, patients in the corset group showed pathological fractures in $8.6 \%$ and in no-corset group in $9.3 \%(p=0.709)$. The univariate and bivariate analyses demonstrated no significant prognostic factor for incidence of pathological fractures in both groups.

Conclusions: In this analysis, we could show for the first time in more than 900 patients, that abandoning a general corset supply in patients with spinal metastases does not significantly cause increased rates of pathological fractures. Importantly, the incidence of pathological fracture after RT was small.
\end{abstract}

\section{Background}

Spinal bone metastases represent the most frequent site of skeletal metastases [1]. The effects of bone metastases are a major concern in everyday clinical practice and result in pain at rest and during activity, limitations in daily life, lower performance ability, risk of pathological fractures and neurologic deficits [2], with a significant reduction in the patients' quality of life (QoL). Radiotherapy (RT) is the most common treatment option of bone metastases in advanced tumor disease [3]. The aim of therapy hereby is to reduce pain, to improve the functionality, and to prevent complications, for example compression of the spinal cord and pathological fractures. Pathologic fractures occurred in $39 \%$ of patients with breast cancer, in $22 \%$ of patients with prostate

\footnotetext{
* Correspondence: harald.rief@med.uni-heidelberg.de

'Department of Radiation Oncology, University Hospital of Heidelberg, Im

Neuenheimer Feld 400, 69120 Heidelberg, Germany

Full list of author information is available at the end of the article
}

cancer, and in $22 \%$ of patients with bone metastases from lung cancer or other solid tumors during 12, 15, and 21 months of follow up, respectively $[4,5]$. Consequently, pathologic fractures are a significant clinical concern in these patient populations, and preventing or delaying fractures is an important treatment objective. In previous retrospective studies among American and Japanese populations, the incidence of pathologic fractures in the vertebral column is estimated to range at $10 \%[6,7]$. Clinical care of unstable metastases in many centers often includes patient immobilization either by means of an orthopedic thoracic corset or by confining the patient to bed in order to prevent pathological fractures, which further decreases patients` QoL. Accordingly the incidence of pathological fractures after RT in patients with spinal bone metastases while wearing an orthopedic corset is still unknown. The aim of this retrospective analysis was to evaluate the incidence of pathological fractures after RT in patients with and without 
orthopedic corsets and to assess prognostic factors for incidence of pathological fractures in patients with spinal bone metastases.

\section{Methods}

A cohort of 915 patients, was treated by RT for osteolytic metastases of the vertebral column due to histologically diagnosed solid tumors at the University Clinic of Heidelberg in the period from January 2000 until January 2012. All patients were examined using computed tomography scans (CT) in this retrospective analysis. Inclusion criteria were an osteolytic phenotype, location in the thoracic or lumbar spine and a minimum duration of follow-up treatment of 6 months. A total of 2.195 bone lesions in the thoracic and lumbar spine were identified. Bone metastases diagnoses were verified by $\mathrm{CT}$. The patient data were taken from the Heidelberg NCT Cancer Registry and are summarized in Table 1. Performance status was expressed using the Karnofsky Performance Score (KPS) [8]. The specifications for an unstable vertebral body were tumor occupancy of more than $60 \%$ of the vertebral body, and pedicle destruction [9]. Patients with an orthopedic corset used a thoraco-lumbo-sacral orthosis (TLSO) brace. The prescribed corset was prophylactically with no relation to existence of a pathological fracture. The pathological fractures were evaluated in the irradiated spinal region. New diagnosed fractures were analyzed prior to RT and 6 months after RT. This study was approved by the Heidelberg Ethics Committee on 22 October 2012 (nr S- 513/2012).

Table 1 Patient characteristics

\begin{tabular}{|c|c|c|c|c|c|c|c|}
\hline \multirow{3}{*}{$\overline{\text { Age (mean, SD) }}$} & & \multicolumn{2}{|c|}{ Corset group } & \multicolumn{2}{|c|}{ No corset group } & \multicolumn{2}{|l|}{ All } \\
\hline & & $n$ & $\%$ & $n$ & $\%$ & $n$ & $\%$ \\
\hline & & \multicolumn{2}{|c|}{$63.2(+/-11.4)$} & \multicolumn{2}{|c|}{$62.2(+/-10.8)$} & \multicolumn{2}{|c|}{$62.7(+/-11.1)$} \\
\hline \multirow[t]{2}{*}{ Gender } & male & 236 & 53.4 & 253 & 53.5 & 489 & 53.4 \\
\hline & female & 206 & 46.6 & 220 & 46.5 & 426 & 46.6 \\
\hline \multirow[t]{2}{*}{ KPS } & $<=70$ & 204 & 46.2 & 218 & 46.1 & 422 & 46.1 \\
\hline & $>70$ & 238 & 53.8 & 255 & 53.9 & 493 & 53.9 \\
\hline \multirow[t]{6}{*}{ Primary site } & NSCLC & 101 & 22.9 & 206 & 43.5 & 425 & 46.5 \\
\hline & Breast & 219 & 49.6 & 74 & 15.6 & 175 & 19.1 \\
\hline & Kidney & 71 & 16.1 & 88 & 18.6 & 159 & 17.4 \\
\hline & Melanoma & 15 & 3.4 & 26 & 5.5 & 41 & 4.5 \\
\hline & Prostate & 4 & 0.9 & 13 & 2.8 & 17 & 1.9 \\
\hline & Other & 51 & 11.5 & 105 & 22.2 & 98 & 10.6 \\
\hline \multirow[t]{2}{*}{ Localization } & Thoracic & 284 & 64.3 & 279 & 59.0 & 563 & 61.5 \\
\hline & Lumbar & 158 & 35.7 & 194 & 41.0 & 352 & 38.5 \\
\hline \multirow[t]{2}{*}{ Chemotherapy } & yes & 242 & 54.7 & 260 & 55.1 & 502 & 54.9 \\
\hline & no & 200 & 45.3 & 212 & 44.9 & 412 & 45.1 \\
\hline \multirow[t]{2}{*}{ Stability before RT } & stable & 140 & 31.7 & 320 & 67.7 & 460 & 50.3 \\
\hline & unstable & 302 & 68.3 & 153 & 32.3 & 455 & 49.7 \\
\hline \multirow[t]{2}{*}{ Stability after 3 months } & stable & 152 & 39.8 & 279 & 79.0 & 449 & 59.2 \\
\hline & unstable & 230 & 60.2 & 79 & 21.0 & 309 & 40.8 \\
\hline \multirow[t]{2}{*}{ Stability after 6 months } & stable & 165 & 45.1 & 311 & 85.2 & 476 & 65.1 \\
\hline & unstable & 201 & 54.9 & 54 & 14.8 & 255 & 34.9 \\
\hline \multirow[t]{2}{*}{ Bisphosphonates } & yes & 351 & 79.4 & 296 & 62.6 & 647 & 70.7 \\
\hline & no & 91 & 20.6 & 177 & 37.4 & 268 & 29.3 \\
\hline \multirow[t]{4}{*}{ Distant metastases } & Brain & 50 & 11.3 & 77 & 16.3 & 127 & 13.9 \\
\hline & Lung & 74 & 16.7 & 118 & 25.0 & 192 & 21.0 \\
\hline & Liver & 70 & 15.8 & 116 & 24.5 & 186 & 20.3 \\
\hline & Skin & 11 & 2.5 & 25 & 5.3 & 36 & 3.9 \\
\hline \multirow[t]{2}{*}{ Number of metastases } & solitary & 189 & 42.8 & 228 & 48.2 & 417 & 45.6 \\
\hline & multiple & 253 & 57.2 & 245 & 51.8 & 498 & 54.4 \\
\hline
\end{tabular}

SD Standard deviation; KPS Karnofsky performance score; RT Radiotherapy 


\section{Statistical analysis}

The empirical distribution of continuous variables is described by the number of observations, mean and standard deviation; the description of categorical variables includes the number and percentage of patients belonging to the relevant categories. We estimated number of observations of pathological fractures before and 6 months after RT and compared them between groups according to the chisquare test. The univariate log-rank test was used to evaluate the prognostic importance for occurrence of pathological fractures of gender, Karnofsky performance score, non-small cell lung cancer (NSCLC), breast cancer, kidney cancer, localization of metastases, chemotherapy prior to RT, stability prior to RT, stability after 3 months, stability after 6 months, bisphosphonates, and number of bone metastases. Results were reported as $p$-values of the logrank tests. Bivariate analysis was performed to detect factors independently associated with pathological fractures using a Cox regression model. This regression analysis was performed including gender (male), Karnofsky performance score $(<=70)$, NSCLC (no primary), breast cancer (no primary), kidney cancer (no primary), localization of metastases (thoracic), chemotherapy prior to RT (no chemotherapy), stability prior to RT (unstable), stability after 3 months (unstable), stability after 6 months (unstable), bisphosphonates (no bisphosphonates), and number of bone metastases (solitary metastasis). The results are reported as $p$-values, odds ratios and $95 \%$ confidence intervals (CI). For all analyses, a $p$-value of 0.05 or less was considered significant. All statistical analyses were done using the SAS software version 9.3 (SAS Institute, Cary, NC, USA).

\section{Radiotherapy}

RT was performed in the Department of Radiation Oncology at the Heidelberg University Clinic. After virtual simulation was performed to plan the radiation schedule, RT was carried out over a dorsal photon field of the 6MV energy range. The photon field covered the specific vertebral body affected as well as the ones immediately above and below. The median individual dose in all patients was 3 Gy (range 2-3 Gy), the median total dose 30 Gy (range 20-35 Gy). The individual and total doses were decided separately for each individual patient, depending on histology, the patient's general state of health, the current staging, and the corresponding prognosis.

\section{Results}

The mean follow-up was 6.3 months for both groups. Of all patients, $31.7 \%$ (140 patients) in the corset group and $67.7 \%$ (320 patients) in the no-corset group were classified as stable prior to RT, 79.4 \% $(n=351)$ and $62.6 \%$ $(n=296)$ of the corset and no corset group were also treated with bisphosphonates. Considering the number of metastases, $57.2 \%(n=253)$ in the corset group and $51.8 \%$ $(n=245)$ in the no corset group showed multiple metastases. The incidence of unstable metastases was higher in the corseted group (68.3\%) compared to the non-corseted group $(32.3 \%)$ prior to RT $(p<0.01)$. The incidence of pathological fractures prior to RT was $7.4 \%$ in all patients. In the corset group, 6.8 and $8.0 \%$ in no-corset group showed pathological fractures prior to RT, no significant difference between groups was detected $(p=0.473)$. After 6 months, the fracture rate was in total $9.0 \%$ for all patients and correspond $1.6 \%$ new diagnosed fractures. Patients in corset group showed in $8.6 \%$ and in no-corset group in $9.3 \%$ pathological fractures (correspond 1.8 and $1.3 \%$ new diagnosed fractures) $(p=0.709)$. The thoracic spine showed more fractures significantly (Table 2).

The univariate and bivariate analyses identified no significant prognostic factors for incidence of pathological fractures in both groups (Tables 3 and 4).

\section{Discussion}

Bone metastases are common in patients with advanced malignancies. The spinal column is the most common site of bone metastases. Metastatic vertebral body collapse is one of the major causes of severe back pain and

Table 2 Pathological fractures before and after RT

\begin{tabular}{|c|c|c|c|c|c|c|c|c|}
\hline & & \multicolumn{2}{|l|}{ All } & \multicolumn{2}{|c|}{ Corset group } & \multicolumn{2}{|c|}{ No corset group } & \multirow{2}{*}{$\begin{array}{l}p \text {-value } \\
\text { between groups }\end{array}$} \\
\hline & & $n$ & $\%$ & $n$ & $\%$ & $n$ & $\%$ & \\
\hline Pathological fracture & yes & 68 & 7.4 & 30 & 6.8 & 38 & 8.0 & \\
\hline \multirow[t]{3}{*}{ before RT } & no & 847 & 92.6 & 412 & 93.2 & 435 & 92.0 & 0.473 \\
\hline & thoracic & 42 & 61.8 & 22 & 73.3 & 20 & 52.6 & \\
\hline & lumbar & 26 & 38.2 & 8 & 26.7 & 18 & 47.4 & 0.081 \\
\hline Pathological fracture & yes & 82 & 9.0 & 38 & 8.6 & 44 & 9.3 & \\
\hline \multirow[t]{3}{*}{ after 6 months } & no & 833 & 91.0 & 404 & 91.4 & 429 & 90.7 & 0.709 \\
\hline & thoracic & 51 & 62.2 & 28 & 73.7 & 23 & 52.3 & \\
\hline & lumbar & 31 & 37.8 & 10 & 26.3 & 21 & 47.7 & 0.046 \\
\hline
\end{tabular}

Pathological fractures before and 6 months after RT in corset and no corset group 
Table 3 Univariate analysis of prognostic factors for incidence of pathological fractures

\begin{tabular}{lll}
\hline Factor & $\begin{array}{l}\text { Corset group } \\
p \text {-value }\end{array}$ & $\begin{array}{l}\text { No corset group } \\
p \text {-value }\end{array}$ \\
\hline Gender & 0.357 & 0.642 \\
KPS & 0.402 & 0.819 \\
NSCLC & 0.101 & 0.184 \\
Breast cancer & 0.496 & 0.091 \\
Kidney cancer & 0.072 & 0.195 \\
Localization & 0.205 & 0.342 \\
Chemotherapy & 0.454 & 0.379 \\
Stability before RT & 0.268 & 0.677 \\
Stability after 3 months & 0.513 & 0.614 \\
Stability after 6 months & 0.202 & 0.402 \\
Bisphosphonates & 0.183 & 0.420 \\
Number of metastases & 0.145 & 0.229 \\
\hline
\end{tabular}

neurologic compromise. Therefore, prevention of pathologic fractures is of clinical importance to maintain patients` QoL.

Bone metastases from solid tumors can dramatically increase bone resorption, resulting in skeletal complications such as pathologic fractures (10-20\% of patients), spinal cord compression (5 \% of patients), hypercalcemia of malignancy (10-15\% of patients), severe bone pain requiring palliative $\mathrm{RT}$, and represent important clinical issues. Fractures may cause severe bone pain, limit mobility, and require surgery and hospitalization for treatment $[10,11]$.

Our results showed a pathological fracture rate of $7.4 \%$ in all patients. First, we compared between orthopedic corset and no-corset groups to examine the effectiveness of the corset for prevention of a pathological fracture. The corset group with 6.8 and $8.0 \%$ in the no-corset group did not differ between groups. Additionally, after 6 months no significant difference between groups was detected. In a recent trial, the results showed a pathological fracture rate in $18 \%$ of the vertebral bodies prior to RT. New fractures up to 6 months after therapy were seen in $2 \%$ of all cases [12]. This 6-months fracture rate was comparable to our results. In previous retrospective studies among American and Japanese populations, the incidence of pathologic fractures in the vertebral column ranges around $10 \%[6,7]$ and corresponds to our findings. In a further analysis by Saad et al. [13], the risk of pathological fracture in association with lung cancer is given at $17 \%$; this finding, however, was made relative to the entire skeletal system. Pathological fractures are a frequently encountered event; fractures of the vertebral body following RT, on the other hand, are rarely reported. In our results, the thoracic spine showed significant more fractures 6 months after RT. However, $61.5 \%$ of metastases were detected in the thoracic spine. The rib cage and sternum can provide additional structural support, but we could not detect any influence to our results. In the clinical treatment of spinal metastases, many advances have been made in the ability to determine both the size and location of vertebral lesions $[14,15]$. Elevated risk of pathologic vertebral body fracture may not, by itself, justify prophylactic stabilization, as many fractured vertebrae are stable or fractured in a manner that does not compromise the spinal canal [2]. Increased tumor size, lower BMD, increased load, and pedicle involvement elevate the risk of burst fracture prior to endplate failure [15]. The study by Taneichi et al. [9] defines the risk factors for fractures of the vertebral bodies caused by osteolytic metastases and rates the estimated fractures according to different types of metastatic involvement, establishing

Table 4 Bivariate analysis of prognostic factors for incidence of pathological fractures

\begin{tabular}{|c|c|c|c|c|c|c|c|c|c|}
\hline & \multicolumn{3}{|l|}{ All } & \multicolumn{3}{|c|}{ Corset group } & \multicolumn{3}{|c|}{ No corset group } \\
\hline & OR & $95 \% \mathrm{Cl}$ & $p$-value & OR & $95 \% \mathrm{Cl}$ & $p$-value & OR & $95 \% \mathrm{Cl}$ & $p$-value \\
\hline Gender & 0.797 & $0.503-1.262$ & 0.333 & 0.727 & $0.369-1.434$ & 0.358 & 0.862 & $0.461-1.612$ & 0.642 \\
\hline KPS & 0.843 & $0.535-1.327$ & 0.461 & 0.753 & $0.387-1.465$ & 0.403 & 0.930 & $0.500-1.731$ & 0.819 \\
\hline NSCLC & 0.604 & $0.376-0.969$ & 0.036 & 0.566 & $0.285-1.126$ & 0.105 & 0.645 & $0.336-1.237$ & 0.186 \\
\hline Breast cancer & 0.562 & $0.284-1.113$ & 0.098 & 0.745 & $0.318-1.746$ & 0.498 & 0.369 & $0.111-1.225$ & 0.103 \\
\hline Kidney cancer & 1.071 & $0.595-1.928$ & 0.818 & 2.008 & $0.928-4.345$ & 0.076 & 0.534 & $0.204-1.398$ & 0.202 \\
\hline Localization & 0.970 & $0.607-1.548$ & 0.897 & 0.618 & $0.292-1.308$ & 0.208 & 1.351 & $0.725-2.517$ & 0.343 \\
\hline Chemotherapy & 1.020 & $0.647-1.609$ & 0.931 & 1.295 & $0.657-2.555$ & 0.455 & 1.329 & $0.704-2.511$ & 0.381 \\
\hline Stability before RT & 0.937 & $0.595-1.474$ & 0.777 & 0.647 & $0.298-1.406$ & 0.271 & 1.155 & $0.586-2.276$ & 0.677 \\
\hline Stability after 3 months & 0.993 & $0.594-1.660$ & 0.980 & 0.776 & $0.363-1.660$ & 0.514 & 1.266 & $0.505-3.174$ & 0.614 \\
\hline Stability after 6 months & 0.939 & $0.537-1.642$ & 0.826 & 0.587 & $0.256-1.343$ & 0.207 & 1.682 & $0.493-5.740$ & 0.406 \\
\hline Bisphosphonates & 0.939 & $0.573-1.538$ & 0.803 & 0.606 & $0.288-1.274$ & 0.186 & 1.313 & $0.676-2.550$ & 0.421 \\
\hline Number of metastases & 1.314 & $0.826-2.090$ & 0.249 & 1.689 & $0.829-3.442$ & 0.149 & 0.683 & $0.365-1.277$ & 0.232 \\
\hline
\end{tabular}


criteria for assessing the risk of vertebral-body fractures. The risk factors for vertebral-body fractures in the thoracic region (T1-T10) are the tumor size and the degree of destruction of the costovertebral joint; in the thoracolumbar and lumbar region (T10-L5), it is the tumor size and degree of pedicle destruction that are the main factors [16]. Therefore, Taneichi et al. [9] conducted radiographic analyses of patients with metastatic spinal tumors and concluded that destruction of the costovertebral joint was one of the major risk factors of vertebral collapse in the thoracic and lumbar spine. Certainly, there are multiple scoring systems to assess spinal instability in the literature $[17,18]$. We used the Taneichi Score because of the practicability and easy appliance in the clinical practice. As a main concern, this scoring system constitutes a simple method for classifying osteolytic metastases in vertebral bodies as "stable"or "unstable"by definition of risk factors such as tumor size and the degree of costovertebral joint destruction for the thoracic region (Th 1 to 10) and tumor size and the degree of pedicle destruction for the lumbar region (Th 11 to L5), which is why this score is employed in this evaluation. Reductions in fracture risk following bisphosphonate treatment are also frequently disproportionate to changes in bone density $[19,20]$. Bisphosphonates were found to reduce the overall risk of skeletal complications by $14 \%$ and to reduce the incidence of fractures by 28 to $37 \%$ [21]. According to our data, both groups had a high bisphosphonates rate so that this bias was negligible small. Recently, however, zoledronic acid has demonstrated efficacy in the management of bone pain and prevention of SREs, including pathologic fractures [19]. However, bisphosphonate therapy was not a prognostic factor for pathological fractures according to our results.

Pathological fractures play a major role in everyday clinical practice. Common clinical care of unstable metastases or existing fractures often includes patient immobilization either by means of an orthopedic corset or by confining the patient to bed in order to prevent pathological fractures, which further decreases patients` quality-of-life (QoL). Secondly, the pain, which can be severe, is mechanical in origin, and frequently the patient is only comfortable when lying still. However, no data regarding the appearance of pathological fractures by wearing a corset exist so far. According to our results, patients without an orthopedic corset did not have an increased risk of pathological fractures after RT. In both groups unstable metastases were detected, while the number in the corset group was significantly higher. In our opinion, wearing a corset also maintains some disadvantages in palliative patients: atrophy of the paravertebral musculature, limitation in mobility and reduction of the QoL. The importance of the corset in stabilityendangering metastases was discussed up to now controversially, whether thereby fractures can be avoided.
The vertical pressure load on the vertebral body also continues with corset, only axial movements can be decreased. Some prognostic factors as mentioned above are already known, our analysis could not identify prognostic factors for both groups. Only no NSCLC as primary site in all patients was significant, however, this result was affected due to a large number of evaluated NSCLC patients. Therefore, this point cannot be derived a factor. The main limitation of this study was the higher incidence of unstable vertebral bodies in patients in the corseted group $(68.3 \%)$ compared to the non-corseted group $(32.3 \%)$. Further limitations were the variety of primary tumors and the exclusion of patients presenting with cervical spine metastases. Among the strengths of our analysis were the large cohort and the very pertinent clinical question for patients with spinal bone metastases. This was, to our knowledge, the very first analysis to determine if a corset had any utility in preventing the incidence of pathologic fractures after routine radiotherapy.

\section{Conclusion}

In this analysis, we could show for the first time in more than 900 patients, that by omission of corsets the fracture rate was not increased. Importantly, the incidence of a pathological fracture after RT was seldom. Large randomized trials are necessary to confirm these findings.

\section{Competing interests}

The authors declare that they have no competing interests.

\section{Authors' contributions}

$H R$ and JD developed and planned this trial. TB is responsible for statistical considerations/basis of the analysis. RF, SR, IS, TBo and HR estimated the fractures of bone metastases. HR made the data collection and was writing the manuscript. All authors read and approved the final manuscript. No funding for this study was provided.

\section{Author details}

1Department of Radiation Oncology, University Hospital of Heidelberg, Im Neuenheimer Feld 400, 69120 Heidelberg, Germany. ${ }^{2}$ Department of Medical Biometry, University Hospital of Heidelberg, Im Neuenheimer Feld 305, 69120 Heidelberg, Germany.

Received: 21 May 2015 Accepted: 15 October 2015

Published online: 20 October 2015

\section{References}

1. Coleman RE. Metastatic bone disease: Clinical features, pathophysiology and treatment strategies. Cancer Treat Rev. 2001;27:165-76.

2. Wyne CM, Hu SS, Lotz JC. Biomechanically derived guidline equations for burst fracture risk prediction in the metastatically involved spine. J Spin Disorders \& Techniques. 2003;16(2):180-5.

3. Gerszten PC, Welch WC. Current surgical management of metastatic spinal disease. Oncology. 2000;14:1013-36.

4. Kohno N, Aogi K, Minami H, Nakamura S, Asaga T, lino Y, et al. Zoledronic acid significantly reduces skeletal complications compared with placebo in Japanese women with bone metastases from breast cancer: a randomized, placebo-controlled trial. J Clin Oncol. 2005;23:3314-21.

5. Saad F, Gleason DM, Murray R, Tchekmedyian S, Venner P, Lacombe L, et al. A randomized, placebo-controlled trial of zoledronic acid in patients with hormone-refractory metastatic prostate carcinoma. J Natl Cancer Inst. 2002;94:1458-68. 
6. Tsuya A, Kurata T, Tamura K, Fukuoka M. Skeletal metastases in non-small cell lung cancer: A retrospective study. Lung Cancer. 2007;57:229-32.

7. Kosteva J, Langer CJ. Incidence and distribution of skeletal metastases in NSCLC in the era of PET. Lung cancer. 2004;46:45.

8. Karnofsky DA, Burchenal JH. The Clinical Evaluation of Chemotherapeutic Agents in Cancer. In: MacLeod CM, editor. Evaluation of Chemotherapeutic Agents. New York: Columbia Univ Press; 1949. p. 191-205.

9. Taneichi H, Kaneda K, Takeda N, Abumi K, Satoh S. Risk factors and probability of vertebral body collapse in metastases of the thoracic and lumbar spine. Spine. 1997;22:239-45.

10. Saad F, Olsson C, Schulman CC. Skeletal morbidity in men with prostate cancer: quality-of-life considerations throughout the continuum of care. Eur Urol. 2004:46:731-9.

11. Coleman RE. Clinical Features of Metastatic Bone Disease and Risk of Skeletal Morbidity. Clinical Cancer Research. 2006;12:6243s-9s.

12. Rief $H$, Bischof M, Bruckner T, Welzel T, Askoxylakis V, Rieken S, et al. The stability of osseous metastases of the spine in lung cancer-a retrospective analysis of 338 cases. RadiatOncol. 2013;8(1):200.

13. Saad F, Lipton A, Cook R, Chen YM, Smith M, Coleman R. Pathologic fractures correlate with reduced survival in patients with malignant bone disease. Cancer. 2007;110(8):1860-7.

14. Whyne CM, Hu SS, Lotz JC. Burst fracture in the metastatically involved spine: development, validation, and parametric analysis of a three-dimensional poroelastic finite-element model. Spine (Phila Pa 1976). 2003;28(7):652-60.

15. Weber $M H$, Burch S, Buckley J, Schmidt MH, Fehlings MG, Vrionis FD, et al. Instability and impending instability of the thoracolumbar spine in patients with spinal metastases: a systematic review. Int J Oncol. 2011;38(1):5-12.

16. Hill T, DÀlessandro P, Murray $K$, Yates $P$. Prognostic factors following pathological fractures. ANZ J Surg. 2015;85:159-63.

17. Fisher CG, DiPaola CP, Ryken TC, Bilsky MH, Shaffrey Cl, Berven SH, et al. A novel classification system for spinal instability in neoplastic disease: an evidence-based approach and expert consensus from the Spine Oncology Study Group. Spine (Phila Pa 1976). 2010;35(22):e1221-9.

18. Fourney DR, Frangou EM, Ryken TC, Dipaola CP, Shaffrey Cl, Berven SH, et al. Spinal instability neoplastic score: an analysis of reliability and validity from the spine oncology study group. J Clin Oncol. 2011;29(22):3072-7.

19. Cummings SR, Karpf DB, Harris F, Genant HK, Ensrud K, LaCroix AZ, et al. Improvement in spine bone density and reduction in risk of vertebral fractures during treatment with antiresorptive drugs. Am J Med. 2002;112(4):281-9.

20. Watts NB, Cooper C, Lindsay R, Eastell R, Manhart MD, Barton IP, et al. Relationship between changes in bone mineral density and vertebral fracture risk associated with risedronate: greater increases in bone mineral density do not relate to greater decreases in fracture risk. J ClinDensitom. 2004;7(3):255-61.

21. McCloskey EV, Guest JF, Kanis JA. The clinical and cost considerations of bisphosphonates in preventing bone complications in patients with metastatic breast cancer or multiple myeloma. Drugs. 2001;61:1253-74.

\section{Submit your next manuscript to BioMed Central and take full advantage of:}

- Convenient online submission

- Thorough peer review

- No space constraints or color figure charges

- Immediate publication on acceptance

- Inclusion in PubMed, CAS, Scopus and Google Scholar

- Research which is freely available for redistribution 